\title{
Roles of microRNA-99 family in human glioma
}

This article was published in the following Dove Press journal:

OncoTargets and Therapy

20 June 2016

Number of times this article has been viewed

\author{
Mingyu Zhang' \\ Yong Guo' \\ Jun $\mathrm{Wu}^{\prime}$ \\ Fenghua Chen' \\ Zhijie Dai ${ }^{2}$ \\ Shuangshi Fan' \\ Pengcheng $\mathrm{Li}^{\prime}$ \\ Tao Song'
}

'Department of Neurosurgery, Xiangya Hospital of Central South University, Changsha, ${ }^{2}$ Institute of Endocrinology and Metabolism, Second Xiangya Hospital, Central South University, Changsha, Hunan, People's Republic of China

Correspondence: Tao Song Department of Neurosurgery, Xiangya Hospital of Central South University, 87 Xiangya Road, Changsha, Hunan 410008, People's Republic of China

Tel/fax +86 73I 89753039

Email songtao97I19@163.com
Objective: Deregulation of microRNA (miR)-99 family members (miR-99a, miR-99b, and miR-100) has been reported to play a crucial role in many cancer types. However, their roles in human gliomas have not been fully elucidated. This study aimed to investigate the expression patterns of miR-99a, miR-99b, and miR-100 in glioma tissues and to evaluate their expression profiles with respect to tumor progression.

Methods: Quantitative real-time polymerase chain reaction was performed to detect the expression levels of miR-99a, miR-99b, and miR-100 in glioma and matched non-neoplastic brain tissues. Then, the associations of their expression with various clinicopathological features of glioma patients were statistically analyzed. Moreover, the roles of miR-99a, miR-99b, and miR-100 in regulating glioma cell migration and invasion were determined via transwell assay in vitro.

Results: Compared with non-neoplastic brain tissues, miR-99a, miR-99b, and miR-100 expression levels were all significantly decreased in glioma tissues (all $P<0.001$ ). miR-99a-low, miR-99b-low, and miR-100-low expression more frequently occurred in glioma patients with low Karnofsky performance score $(<90)$ and high World Health Organization grade (III-IV). Further functional experiments revealed that the enforced expression of miR99a, miR-99b, and miR-100 resulted in the inhibition of cellular migration and invasion in glioma cells.

Conclusion: Our results strongly suggest that the aberrant expression of miR-99a, miR-99b, and miR-100 may be a common feature in human gliomas with aggressive clinicopathological features and may participate in malignant phenotypes of the tumors. These findings highlight the potential of the three miR-99 family members as novel therapeutic targets for human gliomas.

Keywords: microRNA-99 family, glioma, clinicopathological features, migration, invasion

\section{Introduction}

Gliomas, the most common type of malignant tumors in the central nervous system, arise from glia cells and often occur in adults. ${ }^{1}$ As one of the most aggressive and lethal human cancer types, gliomas are categorized as grade I-IV according to clinicopathological criteria of the World Health Organization (WHO). ${ }^{2}$ Low-grade gliomas are regarded as benign and often curable with thorough surgical resection, and seldom have the possibility of evolving into advanced grade lesions. High-grade gliomas are commonly characterized by rapid progression with a poor clinical outcome. ${ }^{3}$ Especially, glioblastoma multiforme (GBM), as WHO grade IV gliomas, are the most aggressive form characterized by unrestrained cellular proliferation, strong resistance to apoptosis, diffuse infiltration, vigorous angiogenesis, and widespread genomic instability. ${ }^{4}$ Growing evidence shows that malignant glioma is a leading cause of death in patients, accounting for $45 \%-55 \%$ of primary intracranial tumors. The annual incidence of malignant glioma is more than five per 100,000 population. ${ }^{5}$ 
With advances in aggressive neurosurgery, radiotherapy, and chemotherapy, the clinical survival rate for glioma has greatly improved..$^{6-8}$ However, the median survival rate of patients with GBM remains only $12-15$ months. ${ }^{9}$ Growing evidence shows a series of complex molecular events underlying the tumorigenesis and tumor progression of gliomas, which remains unclear. ${ }^{10}$ Therefore, it is of great clinical significance to understand the molecular mechanisms involving glioma development, progression, and prognosis, which will be necessary to identify novel therapeutic targets and develop therapeutic strategies for patients with gliomas.

MicroRNAs (miRs), a family of endogenous, small, noncoding RNA molecules with a length of 18-25 nucleotides, regulate the gene expression at a posttranscriptional level by binding to the $3^{\prime}$-untranslated regions of the corresponding target mRNAs, leading to inhibition of translation and/or mRNA degradation. ${ }^{11}$ Each miR often has hundreds of target mRNAs, and more than one-third of human genes may be regulated by miRs. ${ }^{12}$ Accumulating studies have reported that miRs play crucial roles in a variety of biological processes, including cellular development, proliferation, differentiation, apoptosis, cell cycle, and metabolism. ${ }^{13,14}$ Pathologically, emerging evidence shows that miRs are novel players in various diseases such as human cancer. Abnormal expression of miRs is commonly associated with the tumorigenesis and progression of various types of cancers. ${ }^{15}$ According to their functions, miRs act either as oncogenes or tumor suppressors with a cancer-specific manner. ${ }^{16}$ In gliomas, especially, a considerable number of studies have indicated that miRs may contribute to glioma tumorigenesis and simulate glioma cell proliferation, angiogenesis, migration, and invasion. ${ }^{17-21}$

miR-99 family, one of the evolutionarily most ancient miR families whose origin dates back to before the bilaterian ancestor, comprises three members, including miR-99a, miR99b, and miR-100. ${ }^{22}$ miR-99a, located at 21q21, has been proved to be frequently repressed in various tumors such as lung cancer, hepatocellular carcinoma, ovarian cancer, bladder cancer, and prostate cancer. ${ }^{23-27}$ Chakrabarti et al ${ }^{28}$ indicated that the antiglioma effects of photofrin-based photodynamic therapy could be strongly augmented by miR-99a overexpression and this novel combination therapeutic strategy should be used for controlling the growth of human p53 wild-type GBMs both in vitro and in vivo. miR-99b, the sequence of which is the predicted human homologue of mouse miR-99b cloned from mouse brain, has been reported to be deregulated in several tumors such as esophageal cancer, non-small-cell lung cancer, hepatocellular carcinoma, pancreatic cancer, colorectal cancer, and endometrial cancer. ${ }^{29-31}$ The involvement of this miR in human glioma remains unknown. miR-100, localized to chromosome 11, has been found to be a diagnostic and/or prognostic marker for human cancer due to its dysregulation and aberrant expression in the tumorigenesis and tumor progression of several types of cancer such as acute myelocytic leukemia, osteosarcoma, esophageal cancer, nasopharyngeal cancer, breast cancer, non-small-cell lung cancer, hepatocellular cancer, pancreatic cancer, bladder cancer, ovarian cancer, cervical cancer, and endometrioid endometrial carcinoma. ${ }^{32-36}$ Alrfaei et $\mathrm{al}^{37}$ revealed that miR-100 expression was downregulated in GBM compared to normal controls, and indicated that miR-100 could suppress tumor cell proliferation and improve survival in GBM animal models.

Although previous studies have revealed that deregulation of miR-99 family members (miR-99a, miR-99b, and miR-100) can play crucial roles in many cancer types, their roles in human gliomas have not been fully elucidated. The present study aimed to investigate the expression patterns of miR-99a, miR-99b, and miR-100 in human glioma tissues and evaluate their expression profiles with respect to tumor progression.

\section{Materials and methods Patients and tissue samples}

The current study was approved by the Research Ethics Committee of Xiangya Hospital and Second Xiangya Hospital of Central South University, Changsha, People's Republic of China. All patients enrolled in this study gave written informed consent. All specimens were handled and made anonymous according to the ethical and legal standards, and were obtained under sterile conditions during surgery.

A total of 100 patients with gliomas were enrolled in this study. The 100 self-paired tumor tissues and non-neoplastic brain tissues were surgically resected from these patients in the Department of Neurosurgery, Xiangya Hospital of Central South University during the period from January 2010 to December 2013. Based on the clinicopathological criteria provided by WHO, 12 patients were WHO grade I with pilocytic astrocytomas, 36 patients were grade II (including 20 patients with fibrillary astrocytoma, ten patients of protoplasmic astrocytoma, and six patients of oligodendroglioma), 42 patients were WHO III (including 32 patients of anaplastic astrocytoma and ten patients of anaplastic oligodendroglioma), and ten patients were WHO IV (all GBM). Other patients' clinical characteristics such as age, sex, tumor size, and Karnofsky performance score (KPS) were collected for 
Table I Associations of miR-99a, miR-99b, and miR-100 expression with clinicopathological characteristics in human gliomas

\begin{tabular}{|c|c|c|c|c|c|c|c|}
\hline $\begin{array}{l}\text { Clinicopathological } \\
\text { characteristics }\end{array}$ & $\begin{array}{l}\text { Total cases } \\
(\mathrm{N}=100), \mathrm{n}(\%)\end{array}$ & $\begin{array}{l}\text { miR-99a-low } \\
(\mathrm{N}=52), \text { n (\%) }\end{array}$ & $P$-value & $\begin{array}{l}\text { miR-99b-low } \\
(\mathrm{N}=50), \mathrm{n}(\%)\end{array}$ & $P$-value & $\begin{array}{l}\text { miR-1 00-low } \\
(\mathrm{N}=58), \mathrm{n}(\%)\end{array}$ & $P$-value \\
\hline \multicolumn{8}{|l|}{ Age (years) } \\
\hline$<50$ & $48(48.00)$ & $25(52.08)$ & NS & $25(52.08)$ & NS & $25(52.08)$ & NS \\
\hline$\geq 50$ & $52(52.00)$ & 27 (5I.92) & & $25(48.08)$ & & $33(63.46)$ & \\
\hline \multicolumn{8}{|l|}{ Sex } \\
\hline Male & $68(68.00)$ & $36(52.94)$ & NS & $33(48.53)$ & NS & $40(58.82)$ & NS \\
\hline Female & $32(32.00)$ & $16(50.00)$ & & $17(53.13)$ & & $18(56.25)$ & \\
\hline \multicolumn{8}{|l|}{ Tumor size (cm) } \\
\hline$<5$ & $70(70.00)$ & $37(52.86)$ & NS & $35(50.00)$ & NS & $40(57.14)$ & NS \\
\hline$\geq 5$ & $30(30.00)$ & $15(50.00)$ & & $15(50.00)$ & & $18(60.00)$ & \\
\hline \multicolumn{8}{|l|}{ KPS } \\
\hline$<90$ & $60(60.00)$ & $22(36.67)$ & 0.01 & $22(36.67)$ & 0.01 & $22(36.67)$ & $<0.001$ \\
\hline$\geq 90$ & $40(40.00)$ & $30(75.00)$ & & $28(70.00)$ & & $36(90.00)$ & \\
\hline \multicolumn{8}{|l|}{ WHO grade } \\
\hline I & $12(12.00)$ & $0(0)$ & $<0.001$ & $0(0)$ & $<0.001$ & $0(0)$ & $<0.001$ \\
\hline ॥ & $36(36.00)$ & $12(33.33)$ & & $10(27.78)$ & & $16(44.44)$ & \\
\hline III & $42(42.00)$ & $30(7 \mid .43)$ & & $30(71.43)$ & & $32(76.19)$ & \\
\hline IV & $10(10.00)$ & $10(100.00)$ & & $10(100.00)$ & & $10(100.00)$ & \\
\hline
\end{tabular}

Note: $N S=$ not significant $(P>0.05)$.

Abbreviations: KPS, Karnofsky performance score; miR, microRNA; WHO, World Health Organization.

statistical analyses. All patients had newly diagnosed gliomas and had received no chemotherapy or radiation therapy before surgery. For quantitative real-time polymerase chain reaction (PCR) assay, all the tissues were frozen immediately after surgery. The clinicopathological characteristics of all 100 patients are summarized in Table 1.

\section{Quantitative real-time PCR}

Total RNA was extracted from the 100 self-paired tumor tissues and non-neoplastic brain tissues by TRIzol Reagent (Thermo Fisher Scientific, Waltham, MA, USA) according to the users' instructions. Reverse-transcribed complementary DNA was synthesized by using miScript II RT kit (Qiagen, Valencia, CA, USA). Relative expression levels of miR-99a, miR-99b, and miR-100 were measured using SYBR green quantitative real-time-PCR with the Stratagene Mx3000P real-time PCR system (Agilent Technologies, Santa Clara, CA, USA) and normalized with U6 small nuclear RNA. Primer sequences used in this study were as follows: for miR-99a, 5'-CGG AAC CCG TAG ATC CGA T-3' (forward) and 5'-CAG TGC AGG GTC CGA GGT-3' (reverse); for miR-99b, 5'-CAC CCG UAG AAC CGA CCU UGC G-3' (forward) and $5^{\prime}$-CGC GAA TTC ACA GTT GCC TAC AGT CA-3' (reverse); for miR-100, 5'-GCG GCA ACC CGT AGA TCC GAA-3' (forward) and 5'-GTG CAG GGT CCG AGG T-3' (reverse); and for U6, 5'-CTC GCT TCG GCA GCA CAT ATA CT-3' (forward) and 5'-ACG CTT CAC GAA TTT GCG TGT C-3' (reverse). PCR reactions for each miR were repeated three times. The fold-change of each miR was calculated using the $2^{-\Delta \Delta \mathrm{Ct}}$ method. ${ }^{38}$ To evaluate the associations between miR expression and various clinicopathological characteristics, the median value of each miR expression level in glioma tissues was chosen as the cutoff point to divide all 100 patients with gliomas into high- and low-expression groups.

\section{Cell culture and transfection}

Two human glioma cell lines (U87 and U251) were purchased from the cell library of the Chinese Academy of Sciences and cultured in Roswell Park Memorial Institute1640 medium (Thermo Fisher Scientific) supplemented with $10 \%$ fetal bovine serum, $2 \mathrm{mM}$ L-glutamine, and $100 \mathrm{U} / \mathrm{mL}$ penicillin-streptomycin mixture at $37^{\circ} \mathrm{C}$ in a humidified atmosphere containing $5 \% \mathrm{CO}_{2}$. The miR-99a mimics, miR-99b mimics, miR-100 mimics, and the corresponding controls (miR-99a-NC, miR-99b-NC, and miR-100-NC) obtained from GenePharma (Shanghai, China) used for the overexpression of miR-99a, miR-99b, and miR-100 in cells were purchased from Ribobio (Guangzhou, China). Cells were seeded into each well of a six-well plate at a final concentration of $2 \times 10^{5}$ cells and transfected for 48 hours using Lipofectamine 2000 Reagent (Thermo Fisher Scientific) based on the manufacturer's instruction.

\section{Transwell assay}

Roles of miR-99a, miR-99b, and miR-100 in the cell migration and invasion abilities were measured using transwell 
inserts with $8 \mu \mathrm{m}$ pores (BD Biosciences, San Jose, CA, USA). For migration assay, $2 \times 10^{5}$ cells were added into the upper chamber with a noncoated membrane. For invasion assay, $2 \times 10^{5}$ cells were added into the upper chamber precoated with matrigel matrix. Roswell Park Memorial Institute-1640 medium supplemented with 10\% fetal bovine serum was added into the lower chambers. After overnight incubation, cells adhering to the upper surface of the membrane were removed and the bottom (migrated or invasive) cells were fixed with $4 \%$ paraformaldehyde and stained with $0.1 \%$ crystal violet to visualize nuclei. The number of migrated or invasive cells was counted under a microscope in five random fields and the means for each chamber were determined. All experiments were repeated three times and data were obtained from three independent experiments.

\section{Statistical analysis}

The software Statistical Package for the Social Sciences Version 13.0 for Windows (SPSS Inc., Chicago, IL, USA) was used for statistical analyses. All data obtained from our experiments were expressed as means \pm standard deviation. The differences among groups were analyzed using
Student's $t$-test. The associations of each miR expression and various clinicopathological characteristics were evaluated by chi-square test. Differences were considered statistically significant when $P$ was $<0.05$.

\section{Results}

\section{Decreased expression of miR-99a, miR-99b, and miR- 100 in glioma tissues}

Compared with non-neoplastic brain tissues, miR-99a (tumor vs normal: $2.67 \pm 1.07$ vs $4.67 \pm 1.13, P<0.001)$, miR-99b (tumor vs normal: $2.57 \pm 0.91$ vs $5.26 \pm 1.19, P<0.001$ ), and miR-100 (tumor vs normal: $2.41 \pm 1.02$ vs $5.74 \pm 0.81$, $P<0.001)$ expression levels were all significantly decreased in glioma tissues (Figure 1).

Then, we divided all patients into miR-99a-low/high, miR-99b-low/high, and miR-100-low/high groups according to our score system. Of 100 patients with gliomas, 52 $(52.00 \%)$ and $48(48.00 \%)$ belonged to miR-99a-low and miR-99a-high groups, respectively; 50 (50.00\%) and 50 (50.00\%) belonged to miR-99b-low and miR-99b-high groups, respectively; and 58 (58.00\%) and 42 (42.00\%) belonged to miR-100-low and miR-100-high groups, respectively.
A

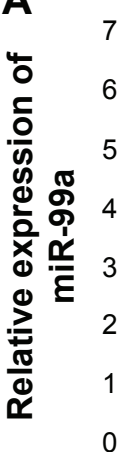

0

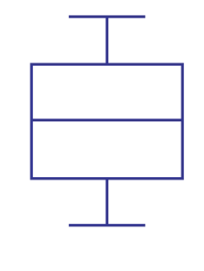

Gliomas

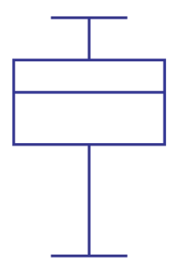

$P<0.001$

Non-neoplastic brain
B

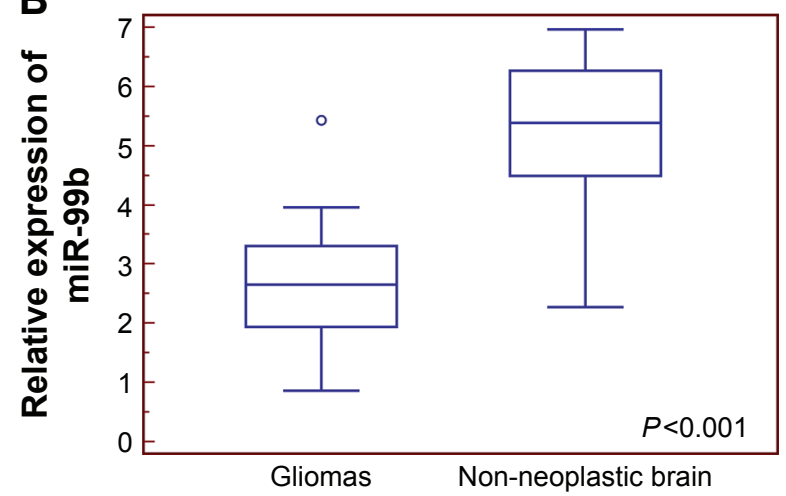

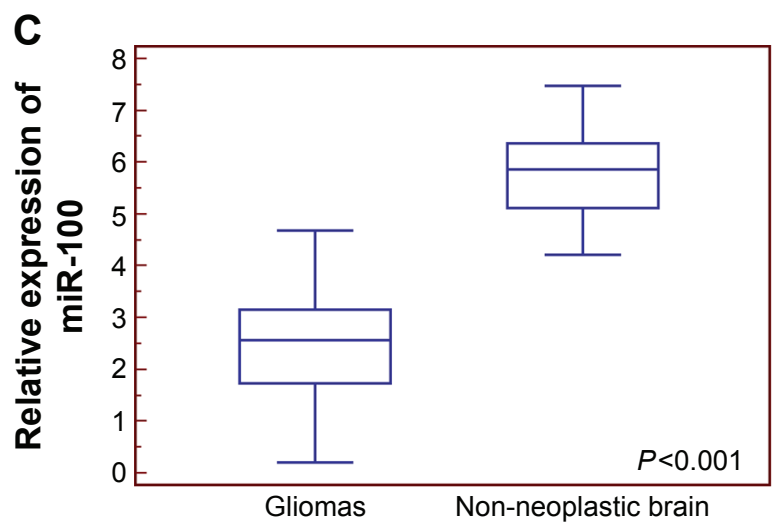

Figure I Relative expression levels of miR-99a (A), miR-99b (B), and miR-100 (C) in human glioma and non-neoplastic brain tissues. Abbreviation: miR, microRNA. 

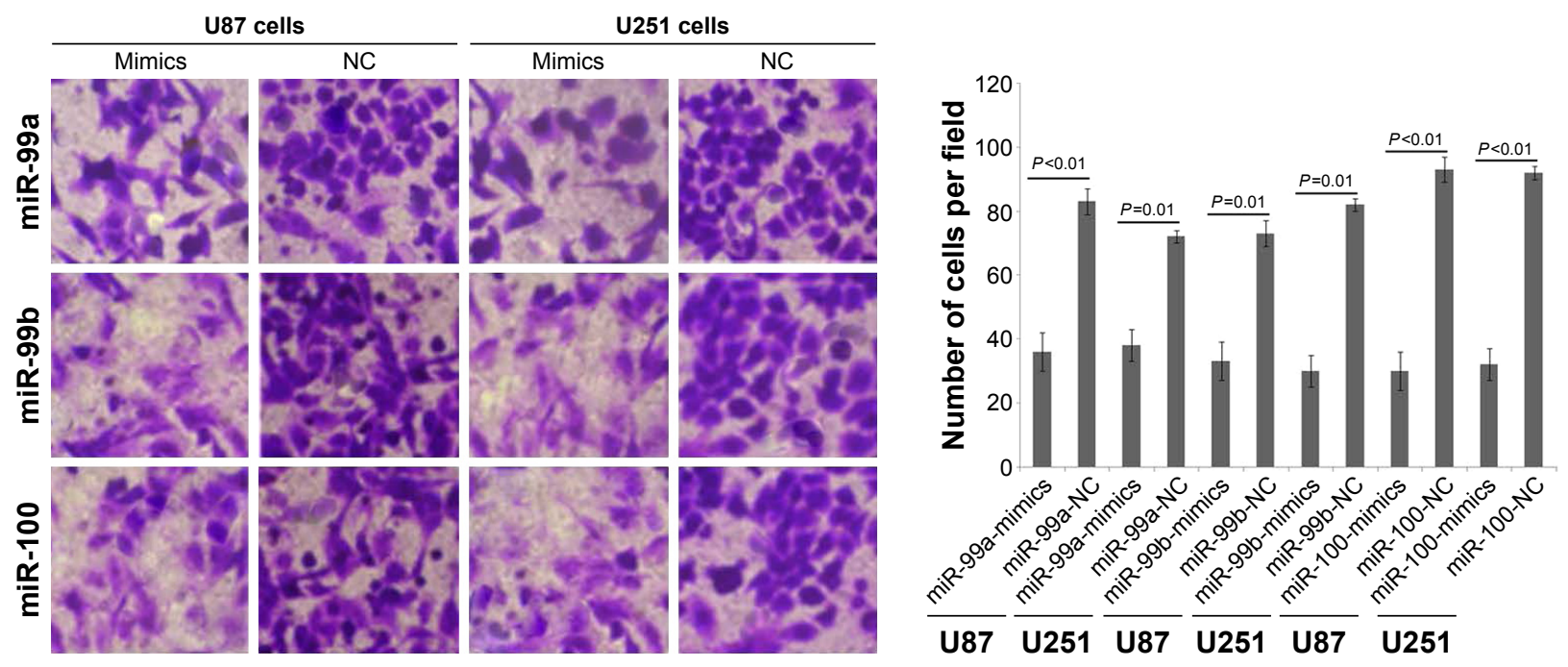

Figure 2 Effects of miR-99a, miR-99b, and miR-100 on migration of glioma cell line.

Notes: Overexpression of miR-99a, miR-99b, and miR-I00 could efficiently suppress the migration abilities of both U87 and U25I cells. Magnification $\times 200$. Abbreviations: miR, microRNA; NC, normal control.

\section{Decreased expression of miR-99a, miR-99b, and miR-I00 associated with aggressive tumor progression of human gliomas}

The associations between miR-99a/99b/100 expression and clinicopathological characteristics were evaluated. As shown in Table 1, miR-99a-low, miR-99b-low, and miR-100-low expression more frequently occurred in glioma patients with low KPS score $(<90)$ and high WHO grade (III-IV) (both $P<0.05$ ). There were no statistically significant differences between miR-99a/99b/100 expression and other clinicopathological characteristics, including patients' age, sex, and tumor size (all $P>0.05$, Table 1).

Enforced expression of miR-99a, miR-99b, and miR- 100 inhibited glioma cell migration and invasion

To determine whether miR-99a, miR-99b, and miR-100 could regulate glioma cell migration and invasion, we performed in vitro glioma cell migration and invasion assays by transfecting miR-99a mimics, miR-99b mimics, and miR-100 mimics into both U87 and U251 cells. As shown in Figures 2 and 3, the overexpression of miR-99a, miR-99b, and miR-100
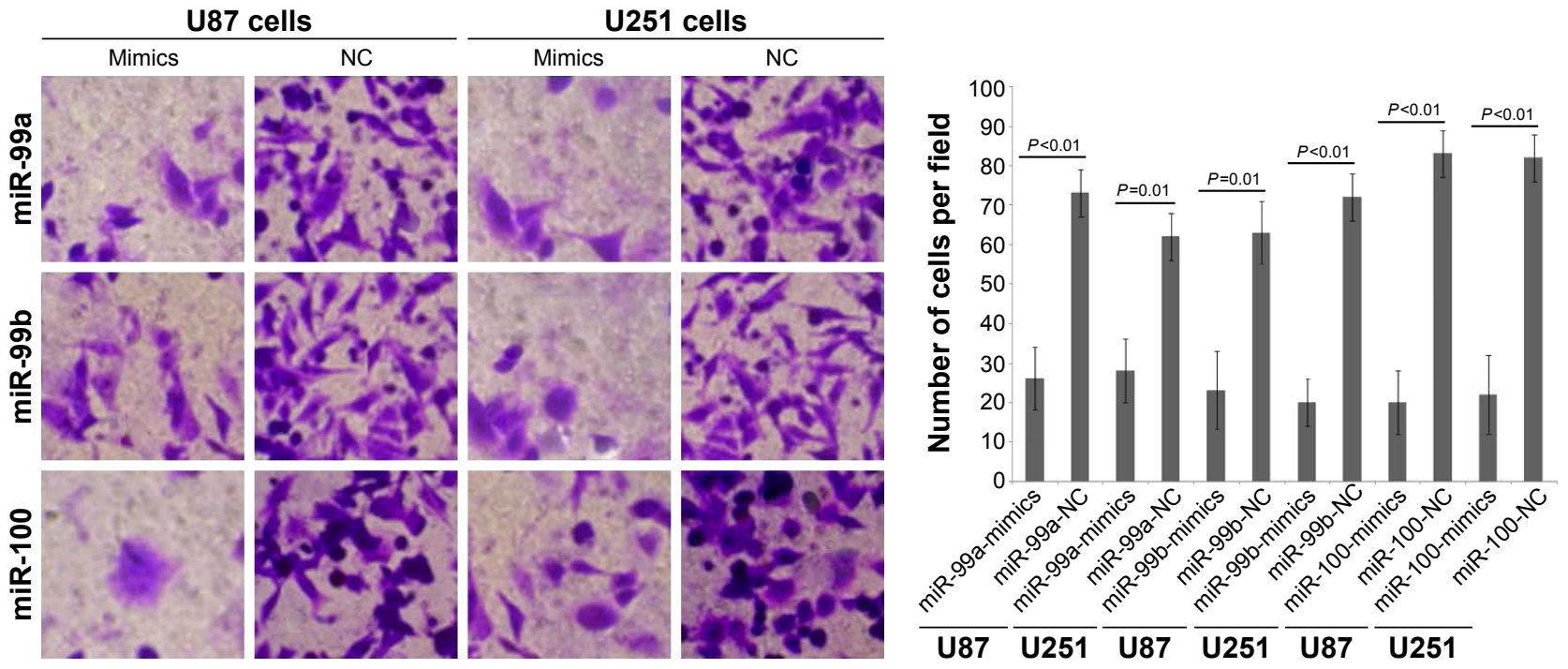

Figure 3 Effects of miR-99a, miR-99b, and miR-100 on invasion of glioma cell line.

Notes: Overexpression of miR-99a, miR-99b, and miR-100 could efficiently suppress the invasion abilities of both U87 and U25I cells. Magnification $\times 200$. Abbreviations: miR, microRNA; NC, normal control. 
could efficiently suppress the migration and invasion abilities of both U87 and U251 cells.

\section{Discussion}

Tumorigenesis and tumor progression of human gliomas are mainly caused by the dysregulation of various genetic risk factors and environmental carcinogenic factors. Accumulating studies have revealed the crucial roles of miRs, a family of highly conserved gene regulators, in malignant changes during the progression of human gliomas. In the present study, we confirmed the aberrant expression patterns of three members of miR-99 family and found their significant associations with aggressive clinicopathological features of patients with gliomas. Functionally, we also demonstrated the tumor suppressive roles of miR-99a, miR-99b, and miR100 in glioma cell migration and invasion. All these results suggest that the miR-99 family members could be used as candidate markers in glioma patients' screening, early diagnosis, and development of novel therapeutic strategies.

The miR-99 family, which is one of the evolutionarily most ancient miR families, functions as a group of crucial facilitators of various disease processes, including cancer. For miR-99a, Wang et $\mathrm{al}^{39}$ performed the gain- and loss-offunction studies and revealed that miR-99a could effectively inhibit breast cancer cell proliferation, migration, and invasion through regulating $H O X A 1$ gene; Huang et $\mathrm{al}^{40}$ reported that miR-99a inhibited tumorigenesis through targeting the mammalian target of rapamycin and its downstream phosphorylated proteins (p-4E-BP1 and p-S6K1) in anaplastic thyroid cancer cells; Chen et $\mathrm{al}^{21}$ indicated that miR-99a was downregulated in human non-small-cell lung cancer and, thus, regulated proliferation, colony formation, and migration through the insulin-like growth factor 1 receptor pathway. For miR-99b, Li et $\mathrm{al}^{31}$ found that the expression level of miR-99b-5p was more than sixfold higher in primary colorectal cancer than in matched liver metastases, and validated the suppressive role of miR-99b-5p in liver metastasis of colorectal cancer; Yang et $\mathrm{al}^{30}$ showed that miR-99b was expressed at high levels in tissues of patients with hepatocellular carcinoma, and reported that the elevated levels of miR-99b predicted poor overall survival as well as diseasefree survival of patients with this malignancy; Kang et $\mathrm{al}^{29}$ identified miR-99b as a tumor suppressor in non-small-cell lung cancer and validated fibroblast growth factor receptor 3 as a direct target gene for this miR. For miR-100, its expression was either downregulated in squamous cell carcinoma of the tongue, glioma, thyroid cancer, breast cancer, epithelial ovarian cancer, and endometrial cancer, or upregulated in esophageal squamous cell carcinoma, pancreatic cancer, and bladder cancer. ${ }^{41}$ From the above studies, it may be concluded that miR-99a, miR-99b, and miR-100 can serve as potential regulators for cancer progression.

Similar to other malignancies, we found in this study that the expression levels of miR-99a, miR-99b, and miR100 were all dramatically reduced in human glioma tissues compared to the normal controls. We also observed that low expression of the three miRs had a negative effect on WHO grade and a positive effect on KPS of glioma patients. Cell migration and invasion are key steps for tumor motility in glioma progression. Here, our in vitro data demonstrated that the transfection of miR-99a, miR-99b, and miR-100 remarkably suppressed cell migration and invasion, providing evidence that the three miR-99 family members may exert tumor suppressive roles in gliomas, although more in-depth studies on the exact molecular mechanisms are necessary.

\section{Conclusion}

Our results strongly suggest that the aberrant expression of miR-99a, miR-99b, and miR-100 may be a common feature in human gliomas with aggressive clinicopathological features and may participate in malignant phenotypes of the tumors. These findings highlight the potential of the three miR-99 family members as novel therapeutic targets for human gliomas. However, there are still several limitations of this study. First, accumulating studies have indicated that gliomas may show significant molecular changes specific to the histological type. Further studies based on a large cohort of glioma samples should be performed to validate our findings here. Second, since miRs modulate pathogenesis by suppressing the corresponding target genes, identification of the target genes of miRs is helpful to understand how aberrant miRs are involved in glioma pathogenesis. Thus, further studies are required to identify the potential target genes for miR-99a, miR-99b, and miR-100 and to validate their regulatory mechanisms in glioma cells.

\section{Acknowledgment}

This work was supported by National Natural Science Foundation of People's Republic of China (No 81200644).

\section{Disclosure}

The authors report no conflicts of interest in this work.

\section{References}

1. Hollon T, Hervey-Jumper SL, Sagher O, Orringer DA. Advances in the surgical management of low-grade glioma. Semin Radiat Oncol. 2015;25: 181-188.

2. Rizzo D, Ruggiero A, Martini M, Rizzo V, Maurizi P, Riccardi R. Molecular biology in pediatric high-grade glioma: impact on prognosis and treatment. Biomed Res Int. 2015;2015:215135. 
3. Alexandru-Abrams D, Jadus MR, Hsu FP, Stathopoulos A, Bota DA. Therapeutic targeting of malignant glioma. Anticancer Agents Med Chem. 2014;14:1075-1084.

4. Ramakrishna R, Pisapia D. Recent molecular advances in our understanding of glioma. Cureus. 2015;7:e287.

5. Ohgaki H. Epidemiology of brain tumors. Methods Mol Biol. 2009; 472:323-342.

6. Stupp R, Mason WP, van den Bent MJ, et al. European Organisation for Research and Treatment of Cancer Brain Tumor and Radiotherapy Groups; National Cancer Institute of Canada Clinical Trials Group. Radiotherapy plus concomitant and adjuvant temozolomide for glioblastoma. N Engl J Med. 2005;352(10):987-996.

7. Oermann E, Collins BT, Erickson KT, et al. CyberKnife enhanced conventionally fractionated chemoradiation for high grade glioma in close proximity to critical structures. J Hematol Oncol. 2010;3:22.

8. Ottenhausen M, Krieg SM, Meyer B, Ringel F. Functional preoperative and intraoperative mapping and monitoring: increasing safety and efficacy in glioma surgery. Neurosurg Focus. 2015;38(1):E3.

9. Paw I, Carpenter RC, Watabe K, Debinski W, Lo HW. Mechanisms regulating glioma invasion. Cancer Lett. 2015;362:1-7.

10. Cohen AL, Colman H. Glioma biology and molecular markers. Cancer Treat Res. 2015;163:15-30.

11. Mishra S, Yadav T, Rani V. Exploring miRNA based approaches in cancer diagnostics and therapeutics. Crit Rev Oncol Hematol. 2016; 98:12-23.

12. Osaki M, Okada F, Ochiya T. miRNA therapy targeting cancer stem cells: a new paradigm for cancer treatment and prevention of tumor recurrence. Ther Deliv. 2015;6:323-337.

13. Romero-Cordoba SL, Salido-Guadarrama I, Rodriguez-Dorantes M, Hidalgo-Miranda A. miRNA biogenesis: biological impact in the development of cancer. Cancer Biol Ther. 2014;15:1444-1455.

14. Valinezhad Orang A, Safaralizadeh R, Kazemzadeh-Bavili M. Mechanisms of miRNA-mediated gene regulation from common downregulation to mRNA-specific upregulation. Int J Genomics. 2014;2014: 970607.

15. Singh TR, Gupta A, Suravajhala P. Challenges in the miRNA research. Int J Bioinform Res Appl. 2013;9:576-583.

16. Palanichamy JK, Rao DS. miRNA dysregulation in cancer: towards a mechanistic understanding. Front Genet. 2014;5:54

17. Rolle K. miRNA Multiplayers in glioma. From bench to bedside. Acta Biochim Pol. 2015;62:353-365.

18. Que T, Song Y, Liu Z, et al. Decreased miRNA-637 is an unfavorable prognosis marker and promotes glioma cell growth, migration and invasion via direct targeting Akt1. Oncogene. 2015;34:4952-4963.

19. Li Y, Xu J, Chen H, et al. Comprehensive analysis of the functional microRNA-mRNA regulatory network identifies miRNA signatures associated with glioma malignant progression. Nucleic Acids Res. 2013; 41:e203.

20. Chen D, Chen Z, Jin Y, et al. MicroRNA-99 family members suppress Homeobox A1 expression in epithelial cells. PLoS One. 2013;8: e80625.

21. Liu N, Tu Y. Systematic review of microRNAs and its therapeutic potential in glioma. Cancer Transl Med. 2015;1:50-66.

22. Li Q, Tu Y. Genetic characteristics of glioblastoma: clinical implications of heterogeneity. Cancer Transl Med. 2015;1:176-180.
23. Chen C, Zhao Z, Liu Y, Mu D. microRNA-99a is downregulated and promotes proliferation, migration and invasion in non-small cell lung cancer A549 and H1299 cells. Oncol Lett. 2015;9:1128-1134.

24. Yang Z, Han Y, Cheng K, Zhang G, Wang X. miR-99a directly targets the mTOR signalling pathway in breast cancer side population cells. Cell Prolif. 2014;47:587-595.

25. Yu SH, Zhang CL, Dong FS, Zhang YM. miR-99a suppresses the metastasis of human non-small cell lung cancer cells by targeting AKT1 signaling pathway. J Cell Biochem. 2015;116:268-276.

26. Feng Y, Kang Y, He Y, et al. microRNA-99a acts as a tumor suppressor and is down-regulated in bladder cancer. BMC Urol. 2014;14:50.

27. Wang L, Chang L, Li Z, et al. miR-99a and -99b inhibit cervical cancer cell proliferation and invasion by targeting mTOR signaling pathway. Med Oncol. 2014;31:934.

28. Chakrabarti M, Banik NL, Ray SK. Photofrin based photodynamic therapy and miR-99a transfection inhibited FGFR3 and PI3K/Akt signaling mechanisms to control growth of human glioblastoma In vitro and in vivo. PLoS One. 2013;8:e55652.

29. Kang J, Lee SY, Lee SY, et al. microRNA-99b acts as a tumor suppressor in non-small cell lung cancer by directly targeting fibroblast growth factor receptor 3. Exp Ther Med. 2012;3:149-153.

30. Yang J, Liu X, Yuan X, Wang Z. miR-99b promotes metastasis of hepatocellular carcinoma through inhibition of claudin 11 expression and may serve as a prognostic marker. Oncol Rep. 2015;34:1415-1423.

31. Li W, Chang J, Wang S, et al. miRNA-99b-5p suppresses liver metastasis of colorectal cancer by down-regulating mTOR. Oncotarget. 2015;6: 24448-24462.

32. Shi DB, Wang YW, Xing AY, et al. C/EBP $\alpha$-induced miR-100 expression suppresses tumor metastasis and growth by targeting ZBTB7A in gastric cancer. Cancer Lett. 2015;369(2):376-385.

33. Luo J, Chen B, Ji XX, Zhou SW, Zheng D. Overexpression of miR-100 inhibits cancer growth, migration, and chemosensitivity in human NSCLC cells through fibroblast growth factor receptor 3. Tumour Biol. Epub 2015 Aug 28.

34. Gong Y, He T, Yang L, Yang G, Chen Y, Zhang X. The role of miR-100 in regulating apoptosis of breast cancer cells. Sci Rep. 2015;5:11650.

35. Bi Y, Jing Y, Cao Y. Overexpression of miR-100 inhibits growth of osteosarcoma through FGFR3. Tumour Biol. 2015;36(11):8405-8411.

36. Yang XD, Xu XH, Zhang SY, et al. Role of miR-100 in the radioresistance of colorectal cancer cells. Am J Cancer Res. 2015;5:545-559.

37. Alrfaei BM, Vemuganti R, Kuo JS. microRNA-100 targets SMRT/ NCOR2, reduces proliferation, and improves survival in glioblastoma animal models. PLoS One. 2013;8:e80865.

38. Ballester M, Castelló A, Ibáñez E, Sánchez A, Folch JM. Real-time quantitative PCR-based system for determining transgene copy number in transgenic animals. Biotechniques. 2004;37:610-613.

39. Wang X, Li Y, Qi W, et al. MicroRNA-99a inhibits tumor aggressive phenotypes through regulating HOXA1 in breast cancer cells. Oncotarget. 2015;6(32):32737-32747.

40. Huang HG, Luo X, Wu S, Jian B. MiR-99a inhibits cell proliferation and tumorigenesis through targeting $\mathrm{mTOR}$ in human anaplastic thyroid cancer. Asian Pac J Cancer Prev. 2015;16:4937-4944.

41. Qin C, Huang RY, Wang ZX. Potential role of miR-100 in cancer diagnosis, prognosis, and therapy. Tumour Biol. 2015;36:1403-1409.

OncoTargets and Therapy

\section{Publish your work in this journal}

OncoTargets and Therapy is an international, peer-reviewed, open access journal focusing on the pathological basis of all cancers, potential targets for therapy and treatment protocols employed to improve the management of cancer patients. The journal also focuses on the impact of management programs and new therapeutic agents and protocols on

\section{Dovepress}

patient perspectives such as quality of life, adherence and satisfaction. The manuscript management system is completely online and includes a very quick and fair peer-review system, which is all easy to use. Visit http://www.dovepress.com/testimonials.php to read real quotes from published authors. 